\title{
The Evidence for Neural Information Processing with Precise Spike-times: A Survey *
}

\author{
Sander M. Bohte (s.m.bohte@cwi.nl) \\ The Netherlands Institute for Mathematics and Computer Science (CWI), \\ Kruislaan 413, 1098 SJ Amsterdam, The Netherlands
}

\begin{abstract}
This paper surveys recent findings in neuroscience regarding the behavioral relevancy of the precise timing with which real spiking neurons emit spikes. The literature suggests that in almost any system where the processing-speed of a neural (sub)-system is required to be high, the timing of single spikes can be very precise and reliable. Additionally, new, more refined methods are finding precisely timed spikes where previously none where found. This line of evidence thus provides additional motivation for researching the computational properties of networks of artificial spiking neurons that compute with more precisely timed spikes.
\end{abstract}

Keywords: spiking neural networks, precise spike timing, neural coding, synchrony coding, temporal coding

\section{Neural Brains Spike}

The human brain consists of a vast number of neurons: estimates range from 10 to 100 billion. These neurons are spread over a number of anatomically different structures, like brain-stem, cerebellum, and cortex. Within each of these structures, different types of neurons exist, with different connectivity-patterns, different typical responses to activity on the inputs, and ultimately different tasks. What all (at least most) of these neurons share, is a network of connections to and from other neurons. Through these connections, neurons communicate with each other (mostly) by transmitting action potentials, or spikes. The mechanisms governing spike generation in a neuron are understood reasonably well: impinging spikes generate a change in the membrane potential of the target neuron, and when this potential crosses some threshold, a spike is (probabilistically) generated (e.g. figure 1); more details can be found in e.g. (Kandel, Schwartz, \& Jessell, 1991).

Although we can argue that at a local level the workings of individual neurons is understood reasonably well, full understanding of the information processing that networks of these spiking neurons engage in - the "neural code" - remains elusive. Rather, with the development of increasingly sophisticated methods in neuroscience, it is becoming

\footnotetext{
* Extended version of the contribution to the 1st International Workshop on the Future of Neural Networks, FUNN 2003, Eindhoven, The Netherlands.
}

(C) 2004 Kluwer Academic Publishers. Printed in the Netherlands. 


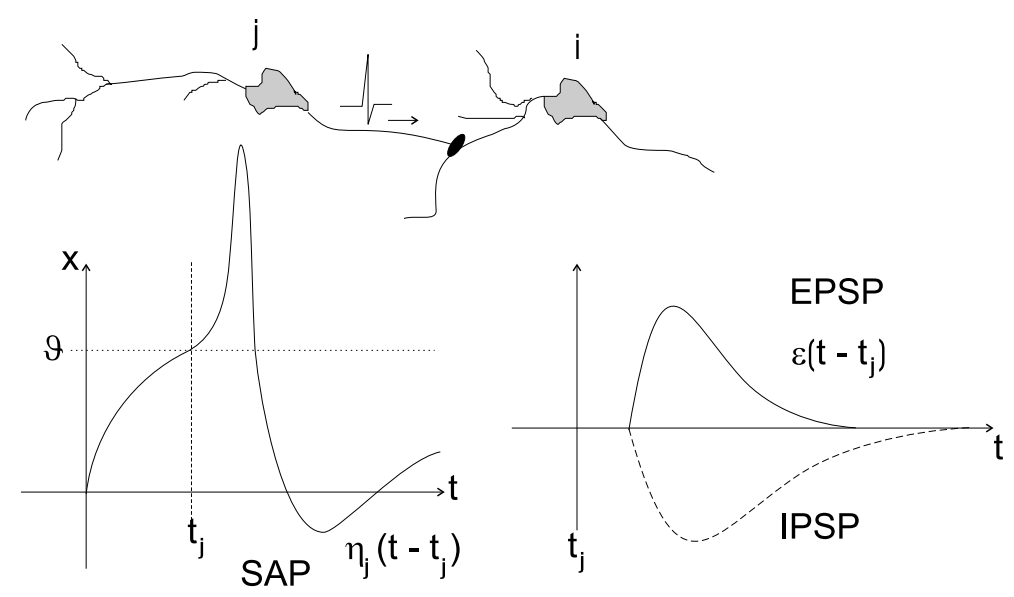

Figure 1. Generation and transmission of spikes: a neuron $j$ generates an action potential when the membrane potential $x$ crosses the threshold $\vartheta$ at time $t_{j}$. After generating a spike, the membrane-potential of neuron $j$ is reset by the spike-after potential (SAP), described by $\eta\left(t-t_{j}\right)$. The spike evokes a response at the postsynaptic neuron $i$ described by the spike-response function $\varepsilon_{i j}\left(t-t_{j}\right)$. The time-course of the voltage response to inhibitory or excitatory synapses is described by the IPSP and EPSP, respectively (after Gerstner (1998)).

clear that the most widely held believe regarding "the neural code" is almost certainly too simply: we now know that neurons do not communicate just by the frequency with which they spike (rate coding). At least part of the information is transmitted in the precise timing of individual spikes. These findings are still quite controversial, and when and how the precise timing of spikes is important for neuronal information processing is a topic of intense debate.

Clearly, precisely timing of spikes would allow neurons to communicate much more information than with essentially random spikes. However, apart from the question whether there is precise spiking, ideas on how such precisely timed spikes can be used are very diverse: from latency coding, where the exact timing of a set of spikes relative to each other contains the information (Maass, 1997b), or, as a variant by rank-coding, where the order of the spikes carries the information (Delorme, Gautrais, VanRullen, \& Thorpe, 1999) (Fig. 2A), to coding by synchrony, where neurons that encode different bits of information on the same object fire synchronously (Fig. 2B), to resonant burst coding, where the frequency of a burst determines which downstream neurons are affected (via resonance) (Izhikevich, Deai, Walcott, \& Hoppensteadt, 2003) (Fig. 2C), and many other variations. Different types of 

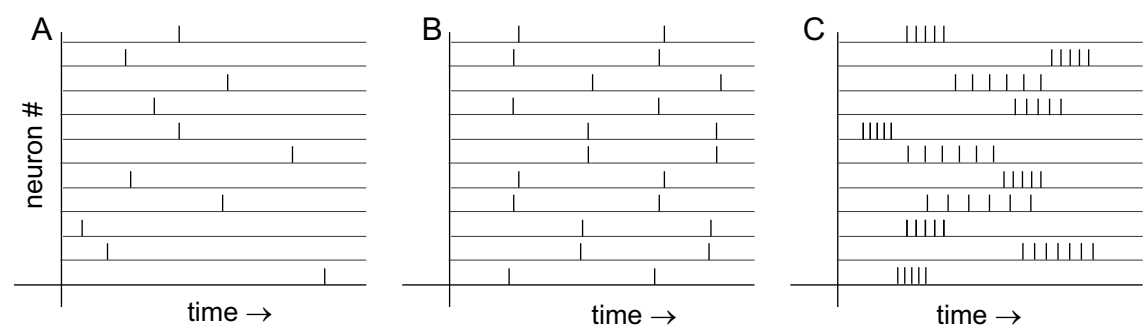

Figure 2. Temporal information coding with precise spike times A) precise spike time patterns over a set of different neurons are the information. Less precise spikes support the notion of rank-coding. B) synchrony coding. Neurons that code information for the same object fire synchronously. C) Burst coding. The frequency of the burst determines which downstream neurons are affected via resonance.

neural coding, including temporal coding, may also coexist on different timescales, e.g. (Oram, Xiao, Dritschel, \& Payne, 2002).

The topic of this paper is a survey of the available neuroscience evidence where precise spike timing is found, and an attempt is made to distill features that these neural systems have in common. Given the accumulated research surveyed, it seems clear that when arguing on behalf of the simple rate-coding scheme for neurons, one now has to demonstrate convincingly beneficial "network externalities" of this scheme, since temporal coding in spiking neurons can achieve most typical neural network tasks much more efficiently. That is, when implemented in spiking neurons, as is the case in the brain. That is not to say that the brain exclusively uses temporal coding, however the final story is clearly not a simple rate-coding one. Additionally, this survey suggests areas and functions of biological neural computation where nature is clearly using the temporal aspect of spiking neural networks, and hence a first direction for building artificial spiking neural networks for practical uses.

\section{Precision and Reliability of Real Spikes}

Whether or not real, biological spiking neurons - or neural systems is general - exhibit a fine temporal precision in their action-potentials is a fundamental question that is much debated but has so far remained unresolved (Bialek, Rieke, Steveninck, \& Warland, 1991; Softky \& Koch, 1993; Shadlen \& Newsome, 1994, 1995; Softky, 1995; Hopfield, 1995; Shadlen \& Newsome, 1998; Mainen \& Sejnowski, 1995; Laurent, 1999; Singer, 1999; Gray, 1999; Shadlen \& Movshon, 1999). Neurophysiological experiments with in vitro cultures of neural tissue have 
shown that the integration process of individual cortical neurons is in principle reliable enough to support precise spike-time coding (Mainen \& Sejnowski, 1995), and here we survey some of the evidence for precise and reliable spiking by real neurons that recent in vivo experiments have uncovered.

When it comes to real, behaving animals, there are specialized subsystems in some animals for which the relevance of temporal information in the spike-times has been clearly demonstrated. Prominent examples are the electro-sensory system of the electric fish (Heiligenberg, 1991), the echolocation-system of bats (Kuwabara \& Suga, 1993), and the auditory system of barn owls (Carr \& Konishi, 1990). In each of these instances, the relative timing of input spikes is used for the computation of the relative direction of the respective sources (AgmonSnir, Carr, \& Rinzel, 1998). Additionally, in the olfactory system of the locust, Laurent et al. have found that odor-recognition is associated with increased spike-time precision (Laurent, Wehr, \& Davidowitz, 1996; Stopfer \& Laurent, 1999), and that modification of the fine temporal structure of spike-trains disrupts the correct odor-classification (Laurent, 1999).

Improving methods. In recent work, DeWeese and Zador (2002) demonstrate precise spike timing in the auditory cortex of the awake behaving monkey: when presenting a fixed tone to the monkey, they describe highly reliable single (binary) spike generation, where these neurons are silent for up to hundreds of milliseconds after generating this one spike. Since the individual neuronal mechanisms cannot account for this "silencing", it almost certainly is a network effect, that is: the cortical networks specifically enforce reliable, binary coding with precisely timed spikes.

An important feature of these findings is that for their measurements, they employ a novel method for recording from in vivo neurons, and they argue that through this method, spikes can be attributed with much higher reliability to specific neurons as compared to traditional multi-unit recordings. With traditional methods, the precise spikes would not have been uncovered.

The Blowfly. In an enlightening study, De Ruyter van Steveninck and Bialek et al. have extensively studied the time-response of the blowfly's motion sensitive neurone H1 during flight (Bialek et al., 1991; de Ryter van Steveninck, Lewen, Strong, Koberle, \& Bialek, 1997; de Ruyter van Steveninck, Borst, \& Bialek, 2001). Bialek et al. have developed a decoding method for reconstructing the (known) original environment as experienced by the blowfly (the input) from the signals measured 
from the H1 neurone. They find that when decoding the signal of $\mathrm{H} 1$, individual spikes contribute significantly to their velocity estimate at each point in time. The accuracy of the decoded signal was found to increase as the spikes were observed with greater temporal precision. In fact, patterns of spikes that differ only by millisecond shifts of the individual spikes could correspond to distinguishable velocity waveforms (Brenner, Strong, Koberle, Bialek, \& de Ruyter van Steveninck, 2000). Furthermore, the timing relationships between neural responses and stimulus events were found to be preserved with millisecond precision, even as signals pass through four stages of neural circuitry. A particular spike, distinguishable in a certain sequence, proved impressively reproducible, with a standard deviation on the onset time of $0.78 \mathrm{~ms}$. The spike directly following is reported to be even more precise, with a standard deviation of only $0.18 \mathrm{~ms}$ (de Ruyter van Steveninck et al., 2001). In fact, the precision of spikes seems to be directly related to the efficiency and noisiness of photoreceptors rather than noisy neurons (Lewen, Bialek, \& de Ruyter van Steveninck, 2001). Bialek et al. remark that the natural environment of the fly while airborne is characterized by signals with high temporal frequencies, and too cope, it seems that the fly's neural systems is efficient up to the physical limits of neural spike-timing.

Summarizing these findings, Fairhall et al. (2001) suggest that neurons in the fly's visual system employ a multilayered coding scheme, where the emitted spikes convey information in several ways: "the timing of individual spikes or short spike-patterns encodes stimulus features that are normalized to the stimulus ensemble, the statistics of interspike intervals on slightly longer timescales encode the stimulus ensemble, and the spike rate can carry information about the changes in ensemble on yet longer timescales." (from Fairhall et al. (2001)).

Vertebrates. In a study of the cat's LGN, Liu et al. (2001) found neural responses that were highly reproducible in their spike timing $( \pm 1-2 \mathrm{~ms})$. They further remark: "This degree of precision only became apparent when an adequate length of the stimulus sequence was specified to determine the neural response, emphasizing that the variable relevant to a cell's response must be controlled to observe the cell's intrinsic response precision." Similarly, Reinagel and Reid (2000) observe high precision in the cat's LGN when subjecting it to stimuli representing "natural scenes", which are typically fast moving sequences of highcontrast images.

Bair and Koch (1996) report that the response of neurons in area MT of the macaque to a repeated stimulus was replicable with high temporal precision $(<2 \mathrm{~ms})$, but only under certain conditions. Bu- 
racas, Zador, DeWeese, and Albright (1998) confirm and extend this finding, and suggest that the high temporal precision can be attributed to the fine temporal structure of the stimulus. The spike-time precision is then of the same magnitude as observed in the rabbit retina (Liu et al., 1997).

A study by Beierholm, Nielsen, Ryge, Alstrom, and Kiehn (2001) adds to this theme: for spikes generated by spinal neurons from the neonatal rat spinal cord, they report a reliable precision of singe spike in the order of $2-3 \mathrm{~ms}$, but only when the input into these cell's has a high frequency and amplitudes $(3-30 \mathrm{~Hz}, 30-200 \mathrm{pA})$. They attribute this somewhat worse temporal precision of spinal neurons as compared to cortical neurons to the broad spikes in the former. Fellous et al. (2001) also report that spike-time reliability is highly dependent on the input frequency, with highest reliability obtained in the frequency range of the typical membrane potential oscillations (4-20 Hz for pyramidal cells in the rat's cortex).

Considerable research is uncovering the nature of the relationship between firing rate and the precise timing of spikes: in particular in the hippocampus the so-called phase-precession phenomenon is observed, i.e. (O'Keefe \& Recce, 1993). Here, the timing of the pyramidal cell spikes relative to the theta rhythm is related to the instantaneous firingrate (and hence position) of the neuron. Roughly, increasing firingrate correlates with earlier spikes (relative to the phase of the theta rhythm. The theta rhythm is an oscillation in the local field potential at about $8 \mathrm{~Hz}$. The precision with which spikes are emitted relative to the theta-phase is remarkable: (Harris et al., 2002) report that in their experiments, mean phase, averaged over all behaviors, shifted from $295^{\circ} \pm 7^{\circ}$ at low instantaneous firing rates $(1$ spike per 2 cycles, $\approx 4 \mathrm{~Hz}$ ) to $141^{\circ} \pm 9^{\circ}$ at high instantaneous firing rates $(\geq 10$ spikes per 2 cycles, $\approx 40 \mathrm{~Hz}$ ). Since the mean is taken as the circular mean $\pm 95 \%$ confidence interval, this means that $95 \%$ of the spikes are fired within $2 \mathrm{~ms}$ and $3.5 \mathrm{~ms}$ respectively to the average phase. It is suggested that the phase-precession phenomenon is an effective means for transforming a rate-code into a temporal code: the proposed underlying mechanism by Mehta, Lee, and Wilson (2002) would faithfully reproduce the temporal order of activation of neurons on short $(<10 \mathrm{~ms})$ time scales. The idea is that this would allow temporal sequence learning by compressing the relevant temporal order of events occurring on long time scales $(>1000 \mathrm{~ms})$, into short times scales $(\approx 10 \mathrm{~ms})$ relevant for synaptic plasticity mechanisms, such as spike-time dependent plasticity (see below). Neural networks inspired by such ideas have been proposed by Hopfield and Brody (2000) for temporal sequence learning. 
Spike-time coding in neural populations. deCharms and Merzenich (1996) report in a study of the macaque's primary auditory cortex that populations of neurons can coordinate the relative timing of their action potentials such that spikes occur closer together in time during continuous stimuli. This way, the neurons can signal stimuli even when their firing rates do not change. deCharms and Merzenich argue that in this fashion, population coding based on relative spike-timing has a number of beneficial properties: the population can systematically signal stimulus features; the signal is topographically mapped; and the signal follows the stimulus time-course even when the mean firing rate does not. They also report similar observations in the awake animal and in the primary somatosensory cortex.

The fact that the rate at which neurons fire does not fully capture the information content conveyed in the spike-train is becoming increasingly well established. This also extends to the idea of population rate-codes like the pooled-response code: this is the proposed means for rapidly transmitting changes in firing-rate via the neural population response, e.g. (Gerstner, 2000). Reich, Mechler, and Victor (2001) recently showed explicitly that the pooled-response code carries significantly less information as compared to the code that keeps track of when which neuron emitted each signal. A significant part of the information is thus transmitted by means other than the firing-rate.

Precise Learning with Spikes. For learning, that is, for adaptively changing synaptic strengths, it has been well established that the temporal order of pre- and postsynaptic spikes can determine synaptic potentiation vs. depression (LTP vs. LTD). A presynaptic spike arriving before a postsynaptic spike is generated tends to lead to potentation of the particular connection; arrival after the postsynaptic spike leads to synaptic depression (Markram, Lübke, Frotscher, \& Sakmann, 1997). Further investigations have revealed that the time-window for such order-dependent synaptic modification is about $20 \mathrm{~ms}$ either way: the arrival of relatively earlier or later presynaptic spikes does not result in LTP or LTD (Bell, Han, Sugawara, \& Grant, 1997; Zhang, Tao, Holt, Harris, \& Poo, 1998; Feldman, Nicoll, Malenka, \& Isaac, 1998; Bi \& Poo, 1998). This phenomenon has become known as spike-timedependent-plasticity (STDP).

A learning rule similar to STDP was already proposed by Gerstner, Kempter, Hemmen, and Wagner (1996) as a means for modeling the learning process that leads to the delay-selection for fine temporal processing by neurons in the auditory system of the barn-owl (as reported by Carr and Konishi (1990)). Such delay selection has also been reported in cultured hippocampal neurons, presumably allowing the 
conversion of temporal information coded in the timing of individual spikes into and stored as spatially distributed patterns of persistent modifications in a neural network (Bi \& Poo, 1999). The issue of any relation between STDP and temporal coding with spiking neurons remains far from resolved (e.g. (Bi \& Poo, 2001; Kepecs, van Rossum, Song, \& Tegner, 2002)), and many believe that STDP may possibly be a by product of learning rules that optimize other neural network properties (e.g. (Chechik, 2003; Eisele \& Miller, 2003; Dayan, Hausser, \& London, 2003)).

\section{Conclusion}

Taking all these data together, it seems clear that the fine temporal structure of impinging spikes, and their impact on the membrane potential, can carry important information, rather than being just noise. Intriguingly, Williams and Stuart (2002) recently reported that distal EPSPs are ineffective sources of background somatic excitation, but, through coincidence detection on the millisecond-scale, have a powerful transient signaling role. The traditional idea that membrane fluctuations should mostly be attributed to the impact of background excitation is thus at least partially challenged.

It is worth noting that the excitement about spiking neural networks did not start with the proposed computational function by Von der Malsburg (1981), but rather by the report of the predicted synchrony or rather: correlations - in the monkey brain by Gray, König, Engel, and Singer (1989). Although the functional relevance of these correlations in terms of dynamic feature binding are now being questioned (e.g Shadlen and Movshon (1999)), these findings, and the availability of ever more sophisticated equipment, have encouraged a close examination of the significance of single spikes in neuronal systems. As demonstrated by the sample outlined above, there are most certainly cases where the timing of single spikes is important. This seems particularly true when the fine temporal structure of the relevant stimuli encountered in the environment is high $(30-300 \mathrm{~ms})$. When neural systems that have to process information from such a fast environment are studied in a much slower one, the temporal precision of single spikes is quickly lost, e.g. (de Ruyter van Steveninck et al., 2001).

The prediction that seems to emerge from the collected evidence would be that fast systems/responses use fast spike-time coding schemes. Tellingly, the human visual system has been shown to be capable of performing very fast classification (Thorpe, Fize, \& Marlot, 1996), where a participating neuron can essentially fire at most one spike. On the 
time scales involved, the relevant input is thus contained in at most one spike per neuron per layer, and, as shown by Stratford, Tarczy-Hornoch, Martin, Bannister, and Jack (1996), even when successive pairs of spikes are transmitted from LGN into the visual cortex, only one spike has a measurable impact on a spiky stellate neuron. The speed involved in decoding auditory information, and even the generation of speech also suggest that most crucial neural systems of the human brain operate quite fast. Very recent research into human fingertip sensory neurons also support this by demonstrating a remarkable precision in the timeto-first spikes from primary sensory neurons (Johansson \& Birznieks, 2004).

Two important questions are now out there: the first is related to methods of neurophysiology, as it seems that increasingly sophisticated methods are uncovering increasingly sophisticated patterns in the spiking behavior of neurons. This almost puts a reverse burden of proof on conventional evidence and conventional wisdom. The second question relates to the future of artificial neural networks: the findings of precise spikes can in principle just mean that the functions of neural information processing traditionally researched with sigmoidal neurons are in fact in some parts of the brain implemented with spiking neurons using reliable spike-timing. The functional equivalence has been amply demonstrated, e.g. (Maass, 1997a; Bohte, Kok, \& La Poutré, 2002b, 2002a). However, as artificial spiking neural networks can be implemented with far fewer biological spiking neurons, the paradigm of rate-coding underlying sigmoidal neurons would have to explicitly compensate this in some way, either through increased (and required) robustness, or through large network effects. The latter idea is of course a challenge to the traditional neural network community. At the same time, the challenge for artificial spiking neural networks is to demonstrate useful functionality that cannot be efficiently implemented using just rate-coding. This is a large order, but answers either way would contribute very significantly to cracking the "neural code".

3.0.0.1. Acknowledgement. Work of SMB in part supported by the Netherlands Organization for Scientific Research (NWO), TALENT grant S-62 588.

\section{References}

AgmonSnir, H., Carr, C., \& Rinzel, J. (1998). The role of dendrites in auditory coincidence detection. Nature, 393, 268-272. 
Bair, W., \& Koch, C. (1996). Temporal precision of spike trains in extrastriate cortex of the behaving macaque monkey. Neural Computation, 8(6), 1185-1202.

Beierholm, U., Nielsen, C., Ryge, J., Alstrom, P., \& Kiehn, O. (2001). Characterization of reliability of spike timing in spinal interneurons during oscillating inputs. J. Neurophysiol., 86, 1858-1868.

Bell, C., Han, V., Sugawara, Y., \& Grant, K. (1997). Synaptic plasticity in a cerebellum-like structure depends on temporal order. Nature, 387, $278-281$.

Bi, B.-q., \& Poo, M.-m. (1999). Distributed synaptic modification in neural networks induced by patterned stimulation. Nature, 401, 792-716.

Bi, G.-q., \& Poo, M.-m. (1998). Synaptic modifications in cultured hippocampal neurons: Dependence on spike timing, synaptic strength, and postsynaptic cell type. J. Neurosci., 18(24), 10464-10472.

Bi, G.-q., \& Poo, M.-m. (2001). Synaptic modification by correlated activity: Hebb's postulate revisited. Annu. Rev. Neurosci., 24, 139-66.

Bialek, W., Rieke, F., Steveninck, R. de Ruyter van, \& Warland, D. (1991). Reading a neural code. Science, 252, 1854-1857.

Bohte, S. M., Kok, J. N., \& La Poutré, H. (2002a). Spike-prop: errorbackpropagation in multi-layer networks of spiking neurons. Neurocomputing, 48(1-4), 17-37.

Bohte, S. M., Kok, J. N., \& La Poutré, H. (2002b). Unsupervised classification in a layered RBF network of spiking neurons. IEEE Trans. Neural Networks, 426-435.

Brenner, N., Strong, S. P., Koberle, R., Bialek, W., \& de Ruyter van Steveninck, R. R. (2000). Synergy in a neural code. Neural Computation, 12(7), 1531-1552.

Buracas, G., Zador, A., DeWeese, M., \& Albright, T. (1998). Efficient discrimination of temporal patterns by motion-sensitive neurons in primate visual cortex. Neuron, 20, 959-969.

Carr, C., \& Konishi, M. (1990). A circuit for detection of interaural time differences in the brain stem of the barn owl. J. Neurosci., 10, 32273246 .

Chechik, G. (2003). Spike-timing-dependent-plasticity and relevant mutual information maximization. Neural Computation, 15, 1481-1510.

Dayan, P., Hausser, M., \& London, M. (2003). Plasticity kernels and temporal statistics. In Nips 2002 (Vol. 15). The MIT Press. (to appear)

de Ruyter van Steveninck, R., Borst, A., \& Bialek, W. (2001). Real-time encoding of motion: answerable questions and questionable answers from the fly's visual system. In J. Zanker \& J. Zeil (Eds.), Motion vision - computational, neural, and ecological constraints. Berlin Heidelberg New York: Springer Verlag. 
de Ryter van Steveninck, R., Lewen, G., Strong, S., Koberle, R., \& Bialek, W. (1997). Reproducibility and variability in neural spike trains. Science, 275, 1805-1808.

deCharms, R., \& Merzenich, M. (1996). Primary cortical representation of sounds by the coordination of action-potential timing. Nature, 381, 610613.

Delorme, A., Gautrais, J., VanRullen, R., \& Thorpe, S. (1999). Spikenet: A simulator for modeling large networks of integrate and fire neurons. Neurocomputing, 989-996.

DeWeese, M., \& Zador, A. (2002). Binary coding in auditory cortex. In Advances in Neural Information Processing Systems (Vol. 15). The MIT Press.

Eisele, M., \& Miller, K. (2003). Hidden markov model of cortical synaptic plasticity: Derivation of the learning rule. In Nips 2002 (Vol. 15). The MIT Press. (to appear)

Fairhall, A., Lewen, G., Bialek, W., \& de Ruyter van Steveninck, R. (2001). Efficiency and ambiguity in an adaptive neural code. Nature, 412, 787792.

Feldman, D., Nicoll, R., Malenka, R., \& Isaac, J. (1998). Long-term depression at thalamocortical synapses in developing somatosensory cortex. Neuron, 21, 347-357.

Fellous, J.-M., Houweling, A., Modi, R., Rao, R., Tiesinga, P., \& Sejnowski, T. (2001). Frequency dependence of spike timing reliability in cortical pyramidal cells and interneurons. J. Neurophysiol., 85, 1782-1787.

Gerstner, W. (1998). Spiking neurons. In W. Maass \& C. M. Bishop (Eds.), Pulsed neural networks (p. 3-55). The MIT Press.

Gerstner, W. (2000). Population dynamics of spiking neurons: Fast transients, asynchronous states, and locking. Neural Computation, 12(1), 43-89.

Gerstner, W., Kempter, R., Hemmen, J. van, \& Wagner, H. (1996). A neuronal learning rule for sub-millisecond temporal coding. Nature, 383, 76-78.

Gray, C. (1999). The temporal correlation hypothesis of visual feature integration: Still alive and well. Neuron, 24, 31-47.

Gray, C., König, P., Engel, A., \& Singer, W. (1989). Oscillatory responses in cat visual cortex exhibit inter-columnar synchronization which reflects global stimulus properties. Nature, 338, 334-337.

Harris, K., Henze, D., Hirase, H., Leinekugel, X., Dragoi, G., Czurkó, A., \& Buzsáki. (2002). Spike train dynamics predicts theta-related phase precession in hippocampel pyramidal cells. Nature, 417, 738-741.

Heiligenberg, W. (1991). Neural nets in electric fish. MIT Press.

Hopfield, J. (1995). Pattern recognition computation using action potential timing for stimulus representation. Nature, 376, 33-36. 
Hopfield, J., \& Brody, C. (2000). What is a moment? transient synchrony as a collective mechanism for spatiotemporal integration. Proc. Natl. Acad. Sci. USA, 97, 13919-13924.

Izhikevich, E., Deai, N., Walcott, E., \& Hoppensteadt, F. (2003). Bursts as a unit of neural information: selective communication via resonance. Trends Neurosci., 26(3), 161-167.

Johansson, R., \& Birznieks, I. (2004). Fist spikes in ensembles of human tactile afferents code complex spatial fingertip events. Nature Neurosci., 7(2), $170-177$.

Kandel, E., Schwartz, J., \& Jessell, T. (Eds.). (1991). Principles of neural science (third ed.). Amsterdam, London, New York: Elsevier/NorthHolland.

Kepecs, A., van Rossum, M., Song, S., \& Tegner, J. (2002). Spike-timing plasticity: common themes and divergent vistas. Biol. Cybern., 87, 446458.

Kuwabara, N., \& Suga, N. (1993). Delay lines and amplitude selectivity are created in subthalamic auditory nuclei: the branchium of the inferior colliculus of the mustached bat. J. Neurophysiol., 69, 1713-1724.

Laurent, G. (1999). A systems perspective on early olfactory coding. Science, 286, 723-728.

Laurent, G., Wehr, M., \& Davidowitz, H. (1996). Temporal representations of odors in an olfactory network. J. Neurosci., 16(12), 3837-3847.

Lewen, G., Bialek, W., \& de Ruyter van Steveninck, R. (2001). Neural coding of naturalistic motion stimuli. Network: Comput. Neural Syst., 12, 317329.

Liu, R., Tzonev, S., Rebrik, S., \& Miller, K. (1997). The structure and precision of retinal spike trains. Proc. Nat. Acad. Sc. USA, 94, 5411-5416.

Liu, R., Tzonev, S., Rebrik, S., \& Miller, K. (2001). Variability and information in a neural code of the cat lateral geniculate nucleus. J. Neurophys., $86,2789-2806$.

Maass, W. (1997a). Fast sigmoidal networks via spiking neurons. Neural Computation, 9(2), 275-304.

Maass, W. (1997b). Networks of spiking neurons: The third generation of neural network models. Neural Networks, 10(9), 1659-1671.

Mainen, Z., \& Sejnowski, T. (1995). Reliability of spike timing in neocortical neurons. Science, 268(5216), 1503-1506.

Markram, H., Lübke, J., Frotscher, M., \& Sakmann, B. (1997). Regulation of synaptic efficacy by coincidence of postsynaptic aps and epsps. Nature, $375,213-215$.

Mehta, M., Lee, A., \& Wilson, M. (2002). Role of experience and oscillations in transforming a rate code into a temporal code. Nature, 417, 741-746. 
O'Keefe, J., \& Recce, M. (1993). Phase relationship between hippocampal place units and the EEG theta rhythm. Hippocampus, 3, 317-330.

Oram, M., Xiao, D., Dritschel, B., \& Payne, K. (2002). The temporal resolution of neural codes: does response latency have a unique role? Phil. Trans. R. Soc. Lond. B, 357, 987-1001.

Reich, D., Mechler, F., \& Victor, J. (2001). Independent and redundant information in nearby cortical neurons. Science, 294, 2566-2568.

Reinagel, P., \& Reid, R. (2000). Temporal coding of visual information in the thalamus. J. Neurosci., 20(14), 5392-5400.

Shadlen, M., \& Movshon, J. (1999). Synchrony unbound: A critical evaluation of the temporal binding hypothesis. Neuron, 24, 67-77.

Shadlen, M., \& Newsome, W. (1994). Noise, neural codes and cortical organization. Curr. Opin. Neurobiol., 4, 569-579.

Shadlen, M., \& Newsome, W. (1995). Is there a signal in the noise? Curr. Opin. Neurobiol., 5, 248-250.

Shadlen, M., \& Newsome, W. (1998). The variable discharge of cortical neurons: implications for connectivity, computation and information coding. J. Neuronsci, 18, 3870-3896.

Singer, W. (1999). Neuronal synchrony: A versatile code for the definition of relations. Neuron, 24, 49-65.

Softky, W. (1995). Simple codes versus efficient codes. Curr. Opin. Neurobiol., 5, 239-247.

Softky, W., \& Koch, C. (1993). The highly irregular firing of cortical cells is inconsistent with temporal integration of random epsp's. J. Neurosci., $13,334-350$.

Stopfer, M., \& Laurent, G. (1999). Short-term memory in olfactory network dynamics. Nature, 402, 610-614.

Stratford, K., Tarczy-Hornoch, K., Martin, K., Bannister, N., \& Jack, J. (1996). Excitatory synaptic inputs to spiny stellate cells in cat visual cortex. Nature, 382, 258-261.

Thorpe, S., Fize, F., \& Marlot, C. (1996). Speed of processing in the human visual system. Nature, 381, 520-522.

von der Malsburg, C. (1981). The correlation theory of brain function. Internal Report 81-2, Max-Planck-Institute for Biophysical Chemistry, Göttingen, Germany.

Williams, S., \& Stuart, G. (2002). Dependence of epsp efficacy on synapse location in neocortical pyramidal neurons. Science, 295, 1907-1910.

Zhang, L., Tao, H., Holt, C., Harris, W., \& Poo, M. (1998). A critical window for cooperation and competition among developing retinotectal synapses. Nature, 395(3), 37-44.

Address for Offprints: Kruislaan 413, 1098 SJ Amsterdam, The Netherlands 
spikeNeuronsNC.tex; 5/02/2004; 14:44; p.14 